EUROPEAN ORGANIZATION FOR NUCLEAR RESEARCH

European Laboratory for Particle Physics

Large Hadron Collider Project

LHC Project Report 339

\title{
THE MAGNETS FOR THE LHC EXPERIMENTS
}

\author{
T.M. Taylor
}

\begin{abstract}
The Large Hadron Collider (LHC) presently under construction at the European Laboratory for Particle Physics (CERN) will provide proton-proton collisions at the $14 \mathrm{TeV}$ level. Each of the four approved detectors (ALICE, ATLAS, CMS, and LHCb) to be installed at the interaction points of this machine relies on a sophisticated magnet system for separation and momentum measurements of the charged particles. The magnets are being designed, manufactured, tested and installed under the technical and financial responsibility of the experiment collaborations, but must satisfy constraints imposed by the laboratory, regarding in particular the cryogenics, powering, controls, and safety. The delivery and assembly schedules are also highly constrained by the requirement to have the magnet systems fully installed before the projected commissioning of the accelerator in 2005. The report will compare the salient features of these magnet systems, and of their integration into the CERN environment.
\end{abstract}

LHC Division

Presented at the 16th International Conference on Magnetic Technology

26 September-2 October 1999 - Ponte Vedra Beach, USA

\footnotetext{
Administrative Secretariat

LHC Division

CERN

CH - 1211 Geneva 23

Switzerland

Geneva, 1 December 1999
} 


\title{
The Magnets for the LHC Experiments
}

\author{
T.M. Taylor \\ CERN, European Organization for Nuclear Research, LHC Division, CH-1211 Geneva 23, Switzerland
}

\begin{abstract}
The Large Hadron Collider (LHC) presently under construction at the European Laboratory for Particle Physics (CERN) will provide proton-proton collisions at the $14 \mathrm{TeV}$ level. Each of the four approved detectors (ALICE, ATLAS, CMS, and $\mathrm{LHCb}$ ) to be installed at the interaction points of this machine relies on a sophisticated magnet system for separation and momentum measurements of the charged particles. The magnets are being designed, manufactured, tested and installed under the technical and financial responsibility of the experiment collaborations, but must satisfy constraints imposed by the laboratory, regarding in particular the cryogenics, powering, controls, and safety. The delivery and assembly schedules are also highly constrained by the requirement to have the magnet systems fully installed before the projected commissioning of the accelerator in 2005 . The report will compare the salient features of these magnet systems, and of their integration into the CERN environment.
\end{abstract}

\section{INTRODUCTION}

A large component of most particle physics experiments is the magnet used to identify and to provide for the measurement of the momentum of the particles emanating from the reaction. The higher the energy of the particles the bigger these magnets need to be. Thus the magnets which will be used for the detectors being built to explore the interactions of the beams of the Large Hadron Collider (LHC), which will be the largest accelerator in the world, are correspondingly large, both in terms of their volume and their stored magnetic energy.

The LHC will provide collisions between counter-rotating beams of $7 \mathrm{TeV}$ protons. In nominal operation the beams consist of bunches of about $10^{11}$ protons spaced at $25 \mathrm{~ns}$ intervals and focused to provide average luminosity of up to $10^{34} \mathrm{~cm}^{-2} \mathrm{~s}^{-1}$. In a typical collision of bunches at this luminosity there will be about 25 events, each $n$ of which gives off about 100 secondary particles, strongly peaked in the forward directions. The big detectors which cover a large fraction of the $4 \pi$ srad solid angle ( $4 \pi$ detectors) investigate up to 200 tracks per bunch crossing, of particles having energies of up to several hundred $\mathrm{GeV}$, and the magnetic field is used for particle identification. In addition, for charged particles the momentum can be measured, either by measuring the sagitta of the trajectory in the magnetic field or by measuring the angle imparted by the field integral. As the LHC will be by far the most powerful accelerator in the world, it was to be expected that the spectrometer magnets should be more powerful than those used in experiments on It is nevertheless important to understand that, while impressive in

Manuscript received September 27, 1999 performance, size and cost, the magnet is but one component of the detector. As the full detector is subject to an overall budget ceiling its geometry and performance is chosen to match in with that of other components [1], and it is useful to recall that

1. the spectrum of particles produced by the interaction is strongly skewed both towards the lower energies and in the direction of the colliding beams;

2. the calorimeters, the resolution of which is inversely proportional to the square root of the energy of the particle, complement effectively the magnet as concerns momentum measurement of high energy particles;

3. too strong a field at the interaction point causes low energy particles to spiral and may complicate the analysis;

4. multiple scattering in the beam pipe and the track detectors sets a limit to the momentum resolution which can be attained.

The LHC detectors have been carefully designed taking these considerations into account, and arrived at very different configurations, in particular the two $4 \pi$ detectors, one of which is based on a solenoid the other (mainly) on toroids. Before describing the actual magnets it is therefore interesting to compare the attributes of magnets for spectrometers in general terms.

\section{The Choice of MAgnets for SPeCtrometers}

The major detectors are particularly interested in analyzing particles having large transverse components of momentum. To be efficient the direction of the field of the spectrometer magnet should subtend a large angle to the associated trajectories, and be independent of the azimuthal angle. It follows that solenoids and toroids are the most appropriate choices for the central region of the experiment. Although it does not provide analyzing coverage over the full azimuth, the transverse field of dipoles (and possibly quadrupoles) finds application in the far forward region because, in contrast with the alternative toroidal geometry, the field volume is free of obstacles which can create confusing background.

Regarding the technology, spectrometer magnets use superconducting coils when performance or economics rule out the resistive option. It is generally admitted that this is the case if the power consumption of a suitable resistive magnet would exceed some 2 to $5 \mathrm{MW}$, depending on the available infrastructure, required duty cycle and coil geometry. As they do not need to be ramped rapidly, these large superconducting coils are usually wound from aluminium stabilized conductor and rely on indirect cooling. 


\section{A. Solenoids}

The vast majority of recent $4 \pi$ detectors have relied on solenoidal type magnets, producing a cylindrically symmetric field having the same axis as the colliding beams. The reason for this is easy to understand: the symmetric 2-D field facilitates reconstruction of the events; there is no material within the field volume to give rise to spurious secondary interactions, and the magnetic forces are relatively easy to contain. In addition, thanks to the large number of magnets that have been made and the experience with their operation, the associated technology is mature.

The sagitta of the trajectory of a charged particle emanating at zenithal angle $\theta$ (i.e. the angle between the trajectory and the axis of the colliding beams) from an interaction on the axis of a long solenoid of radius $\mathrm{R}$ and producing a magnetic field $\mathrm{B}$, is proportional to $\mathrm{BR}^{2} / \sin \theta$. The change of angle is proportional to BR. The "analyzing power" depends on the layout of the detector and is proportional to some combination of measurements of sagitta and changes in angle; this is reduced at small $\theta$ because of the finite length $L$ of the solenoid. Within the limits of known technology and transportable size, the cost of the solenoid is roughly proportional to $\mathrm{LR}^{2} \mathrm{~B}^{2}$. It is therefore clear that, as concerns resolution, it is preferable to invest in size than in central field.

The basic technology for building these large solenoids is mature, so it is relatively easy to design one for incorporation into a given detector. The coils are wound on the inside of a cylindrical aluminum alloy former and the radial magnetic force is taken by the winding itself, in combination with the cylinder. The coil is indirectly cooled by supercritical helium circulating in tubes welded to the cylinder. This geometry is also adapted to making "thin" magnets through which particles pass with little likelihood of interacting, making for clean reconstruction of the events. The solenoids for the LHC differ from previous solenoids, however, in that, due to higher fields, there is higher stress in the winding and it has been necessary to strengthen the conductor.

The effect of the solenoid on the circulating beams is small but must not be neglected. The accelerator has to be provided with skew quadrupoles to correct for the coupling of vertical and horizontal betatron oscillations, and the orbit correction scheme must provide for compensating the effect of a finite crossing angle of the beams.

\section{B. Toroids}

In theory a toroidal magnetic field is ideal for a $4 \pi$ detector at a colliding beam facility. The field is symmetric and perpendicular to the particle motion. The integrated field along the trajectory of a particle increases as $\theta$ decreases which is favorable because the likelihood of having high energy particles to analyze also increases as $\theta$ decreases. Charged particles produced on the axis are deflected in a plane. There is no iron yoke. And there is no field along the axis of the beams.
For an ideal toroid contained between current sheets at radii $\mathrm{R}$ and $\mathrm{r}$ and with field $\mathrm{B}$ at inside radius $\mathrm{R}$ the resolution obtained by combining a measurement of angle before the particle enters the toroidal field, and sagitta within the field, is roughly proportional to $\mathrm{BR} \ln (\mathrm{r} / \mathrm{R}) / \sin \theta$. In this idealized case the optimum resolution is obtained with $\mathrm{R} / \mathrm{r} \approx 3.5$, and is reduced by a factor of about $\sqrt{ } 2$ when $R / r$ is reduced to 2 . The interest for small and intermediate $\theta$ is evident.

So why have we seen so few toroids? It is not (only) because the toroid is a harder magnet to build (which it is). It is mainly because of the difficulty of making in practice anything resembling an ideal toroid, i.e. to make the inner conductor sheet and its supporting structure sufficiently transparent, or to divide it up so as to cover a sufficiently small proportion of the azimuth. This material creates confusion in that some particles are absorbed or interact with the structure, an effect that can outweigh the benefits of conceptual elegance. Various studies made over the last 25 years [1,2] have concluded that the best practical approximation for a high field (and therefore superconducting) toroid, covering the central region of the detector, consists of eight lumped coils. With care these can be made to cast shadows over as little as $30 \%$ of the azimuth, which can be considered acceptable. It implies, however, that a significant fraction of the central detector should lie within the inner radius of the toroid.

In point of fact the catalogue of advantages of the toroid must be read with circumspection. Iron yokes double as calorimeters; the compensation of the effects of solenoids on the circulating beams is fully understood and is not onerous; and for a lumped racetrack toroidal coil geometry the field is far from symmetric. Moreover, the field on the inside conductor of a typical lumped toroid is about four times the maximum field in the useful part of the magnet (compared with about 1.3 times for a solenoid), which is obviously unfavorable in the case of a superconducting coil.

\section{Dipoles (and Quadrupoles)}

While dipoles are not appropriate for the central part of $4 \pi$ detectors, they are the magnets of choice for dedicated "forward" detectors that concentrate on the cone of particles emanating in the forward direction, up to $\theta \approx 300 \mathrm{mrad}$. These magnets are quite challenging. Because of the large aperture and short length the field is highly 3-dimensional, and in order to control the stray field the geometry of the yoke and coils needs to be carefully studied. In addition, a dedicated compensation scheme must be provided for the circulating beams. The preferred saddle shape for the coils is much easier to achieve with a resistive than with a superconducting coil. Unless a suitable geometry of the field can be achieved using either circular or eventually racetrack coils, it is unlikely that any experiment in today's era could afford a superconducting version. In order to maximize the integrated bending field, and to minimize the stray field, it is becoming customary to use sloping poles to marry in with the required acceptance. 
Alternatives to the dipole for the forward region are the quadrupole and the toroid. The toroid provides better acceptance than the dipole, but suffers from its contribution to background through multiple scattering. It does not require compensation. The quadrupole is open and is easier to compensate than the dipole, but the field reduces with reducing $\theta$, and would usually need to be complemented downstream with a dipole.

\section{SPECTROMETER MAGNET SySTEMS FOR THE LHC}

Many details pertaining to these magnets are given in a large number of papers presented at this conference, so for this comparative overview it will be sufficient to concentrate on the salient features of the magnets and on some of their more unusual aspects.

\section{A. $C M S$}

CMS is an acronym for Compact Muon Solenoid. The solenoid can be considered compact in that its diameter is such that the coil can be transported in sections by road. The desired resolution is achieved by increasing the field to more than double that used in such magnets to date.

The detector features a superconducting solenoid having a clear bore of diameter $6 \mathrm{~m}$ and magnetic length of $12.5 \mathrm{~m}$, that has been designed to provide a central field of up to $4 \mathrm{~T}$. At this field level the total stored energy is $2.6 \mathrm{GJ}$. This is more than an a order of magnitude greater than that of the largest solenoids used in (collider) high energy physics experiments to date. The coil will be wound in sections in industry, and transported to CERN where it will be assembled in the surface building of point 5 . The cold mass of the complete coil is 225 $\mathrm{t}$. The flux return yoke consists of a $6000 \mathrm{t}$ barrel section and two $2000 \mathrm{t}$ removable end caps. These are built up of assemblies of steel plates between which are mounted detectors. The magnet will be tested at CERN, and then lowered into place in the underground cavern [3].

Due to the high level of field, the local force on the conductor, which is a NbTi cable co-extruded with pure aluminium stabilizer, is such that it has had to be reinforced with aluminium alloy. This is the major innovation in this magnet. After the co-extrusion process, the flanges of AA6082 aluminium are electron-beam welded without filler to either side of the soft, pure aluminium. The process of co-extrusion of this large conductor and the subsequent welding operation have been the subject of extensive R\&D over many years [4]. The winding of the stiff conductor has also been the object of a pre-industrialization program that has established the feasibility of the process.

\section{B. ATLAS}

The ATLAS experiment features no less than four large superconducting magnets: a central solenoid, a barrel toroid and two end-cap toroids [5]. The overall volume of the system is $8000 \mathrm{~m}^{3}$.
1) Central Solenoid: The central Solenoid has a warm bore of diameter $2.3 \mathrm{~m}$ and length of $5.3 \mathrm{~m}$. At the design value of the central field of $2 \mathrm{~T}$ the stored energy is $39 \mathrm{MJ}$. The 5.5 tonne coil is built to be transparent, and has an equivalent radial thickness of 0.66 radiation lengths [6].

The technology of this "thin" solenoid in extrapolated from previous magnets having similar requirements, but it also incorporates exciting new technology regarding the mechanical characteristics of the conductor. As in the CMS solenoid, the stress level in the conductor is too high to allow the straightforward use of a conductor formed by co-extrusion of a superconducting cable in a stabilizing sheath of pure aluminum. The important characteristic of pure aluminum is its very high RRR (about 1000); its mechanical characteristics are however abysmally poor with a yield strength of about 25 Mpa. For construction alloys, such as A5083, typical values are 5 and $200 \mathrm{MPa}$ respectively. A patient R\&D program has however revealed that by doping the aluminium with a small amount of zinc the stabilizer can be turned into material having usable mechanical characteristics (yield strength > $85 \mathrm{MPa}$ ), and yet keeping the RRR at around 500. More recently it has been found that by using nickel even better performance can be achieved, and the aluminium of the conductor which has been produced for the solenoid has a yield strength of over $100 \mathrm{MPa}$ [7].

The conductor has been by two companies in quantities sufficient to make the coil, which has now been wound and cured, and will be tested next year.

2) Barrel Toroid :The most striking feature of this air-cored toroid is its size. With its inner bore of $9.5 \mathrm{~m}$, outer diameter of $20 \mathrm{~m}$ and length of $26 \mathrm{~m}$, the Barrel Toroid (BT) will be by far the largest magnet ever built. The stored energy of the magnet is 1 GJ. Each of the eight racetrack coils is contained in a massive rigid casing to withstand the magnetic forces. The coil is kept cool by the flow of supercritical helium through a system of tubes attached to the casing. The cold mass of each racetrack is $100 \mathrm{t}$. The conductor consists of a cable similar to that of CMS, co-extruded with pure aluminium. The local force on the conductor is such that it does not need any reinforcement. The R\&D on this magnet has consisted of the construction and test of a $2.7 \mathrm{~m}$ long racetrack coil, and continues with that of the so-called B0 coil. The B0 coil, which is one-third of the length of that of the definitive coils, should be tested at the end of the year. This work has permitted to validate certain technical choices [8]. A considerable effort has also gone into verifying that all conceivable fault conditions are catered for safely. Each coil has its own insulating vacuum enclosure, and will be individually tested at CERN before assembly of the magnet in the underground cavern, where the first test of the complete magnet will take place.

3) End-Cap Toroids:The magnetic spectrometer for ATLAS is completed with the two End Cap Toroids (ECT). These magnets nest into the ends of the BT providing coverage for forward muon spectroscopy. The eight indirectly-cooled coils 
are supported by a lightweight frame, the whole being contained in a single vacuum enclosure. The inner bore of the toroid has a diameter of $1.65 \mathrm{~m}$, the outer diameter is $10.7 \mathrm{~m}$ and the axial length is $5 \mathrm{~m}$. The total mass of each ECT is 239 $\mathrm{t}$ of which $160 \mathrm{t}$ is cold mass, and the stored energy is $250 \mathrm{MJ}$. The field is enclosed within the walls of the cryostat, so only changes of angle of the trajectories can be measured (no sagitta). The engineering of this device has been challenging, and a number of interesting solutions are being incorporated [9]. In particular a significant amount of work went into verifying that the asymmetric forces between the BT and the ECT in certain fault scenarios could be safely contained.

4) System Integration: It is clear that the ATLAS experiment uses a system of magnets, and whereas initially the three types of magnets were treated as separate items, it has become evident that considerable savings can be made by integration, and, in view of the tight budgetary constraints, this is being implemented wherever possible [5]. For example, if found to be feasible, powering the BT and ECT in series could lead to substantial savings.

\section{ALICE}

The principal spectrometer magnet for the ALICE experiment is a resistive solenoid that is at present being used for the L3 experiment at LEP [10]. The main goal of ALICE is to study the interactions of heavy ions (also foreseen in the LHC), and the solenoid of length $12 \mathrm{~m}$ and clear bore diameter $12 \mathrm{~m}$, which produces a field of up to $0.5 \mathrm{~T}$, has been found to be more than adequate. It will in fact be run at $0.2 \mathrm{~T}$, at which level it consumes a modest 1.5 MW. In addition, it is now planned to provide the detector with a muon arm, requiring a large dipole. The conceptual design of this magnet, which features resistive saddle-shaped coils and sloping poles, has been made at CERN, and the engineering is presently under study at JINR, Dubna [11]. The coils are made from hollow aluminium conductor and consume 3.7 MW when the magnet is powered to give its nominal integrated bending strength of 3 Tm. The mass of the magnet is $835 \mathrm{t}$.

The unshielded dipole field also acts on the circulating beams, and requires a local 3-magnet correction scheme.

\section{D. $\mathrm{LHCb}$}

The LHCb experiment focuses on the observation of evidence for $\mathrm{C}_{\mathrm{p}}$ violation, and comprises a single arm spectrometer that also makes use of a large dipole magnet. The technical proposal for this experiment assumed the presence of a superconducting window frame magnet [12]. Such a geometry is attractive because it provides a very uniform field, but a number of engineering studies have shown that its cost would be beyond the means of the collaboration. If it were to be superconducting, the magnet should use racetrack coils, in which case the field is less uniform and a more massive yoke is required to cope with the stray flux. There is however sufficient installed power at LHC point 8, where this experiment is to be installed, and it has now been decided to follow the example of ALICE and concentrate on a resistive magnet, also featuring saddleshaped coils and sloping poles.

In its present design the magnet provides a total integrated field of $4 \mathrm{Tm}$. The pole gap is 2.2 to $3.5 \mathrm{~m}$ vertically (the direction of the field), and 2.6 to $4.2 \mathrm{~m}$ horizontally. The overall length of the magnet (in the beam direction) is $5 \mathrm{~m}$ and its total weight about $1500 \mathrm{t}$. The power dissipation in the aluminium coils will be $4.2 \mathrm{MW}$ [13].

\section{INFRASTRUCTURE}

\section{A. Civil Engineering and General Infrastructure}

The civil engineering associated with the LHC experiments is in full swing. The large shafts and caverns are being excavated, and the surface buildings are being constructed. As regards the magnets, the surface building at point 5 , in which the CMS magnet will be assembled and tested, is advancing rapidly and should be available on schedule to receive components in June 2001. The other work is also progressing well. The caverns are being dug around the existing LEP/LHC tunnel in such a way as to leave it intact and stable, permitting operation of the Large Electron Positron Collider (LEP) until October 2000. At that time LEP will stop and the tunnel will be broken to allow full excavation of the large underground halls which will house the ATLAS and CMS experiments.

Once the construction work is done, CERN will equip the shafts and caverns with power, ventilation and other general services. The cavern at point 1 is the most urgent, as the complex magnet system will be assembled directly in place and tested there, and this will have to be done before installing the rest of the experiment: delivery to the ATLAS experiment collaboration is foreseen for summer 2002. The cavern for CMS is less urgent, and will be delivered in autumn 2003.

\section{B. Cryogenics}

Whereas the magnets and proximity cryogenics are being provided by the experiments, under their responsibility, the Laboratory will have to operate and maintain the cryogenics to cool the magnets, and these installations are being specified and supplied by CERN, in collaboration with the magnet designers $[14,15]$. In this way common solutions are being adopted which will minimize the spares count and make it possible to provide routine operation and maintenance with a minimum of staff.

\section{Follow-UP, COST AND TIME}

These magnets have been designed and are being procured under the responsibility of the collaborations that form the experiment teams. CERN has put at the disposal of these collaborations a few experienced scientists and engineers to pilot the undertaking and to ensure that the magnets satisfy local requirements and fit in with the infrastructure. As the 
magnet is a large cost component (20 to $30 \%$ of the cost of the whole experiment), the collaborations have had frequent reviews and have created oversight boards with the intention of following closely the manufacture and intervening if necessary to ensure that time and cost goals are met. It is vital that these magnets be installed before the start of LHC commissioning, scheduled for the second half of 2005, as later installation would require a very long shutdown of the accelerator shortly after its start up - hardly an attractive proposition. For this reason the CERN LHC Experiments Committee also follows the progress of the magnets through the Magnet Advisory Group.

With regard to purchasing, the collaborations forming the two big experiments have adopted quite different approaches. In CMS the Common Fund of the experiment is used to pay for the components via competitive tender. Whereas some parts are coming in more expensive than foreseen, others are cheaper and the global cost is appearing to be very close to the estimate. In the case of ATLAS the magnets are mainly the object of in-kind contributions to the experiment from various parts of the collaboration, with their value to the experiment stated as being the estimated value, thus transferring the risk of cost overrun to the in-kind contributor. The idea is that everyone wants the experiment to work well and be on time, so if there is a problem they will be highly motivated to solve it. In this way both experiments have so far managed to contain their commitments to the estimated cost. This is good news, as the collaborations are under severe financial constraints, and it was not possible to accord significant contingency for the construction of these magnets.

Concerning how the projects are advancing with respect to time, great progress has been made over the last two years.

Most encouraging has been the successful co-extrusion of the first full lengths of the large conductors, and the completion of the ATLAS inner solenoid winding. The successful testing of the racetrack has also been reassuring in that it has demonstrated the stability of the winding when constrained in the form required for the toroid. If there are no serious problems the magnets could be on time - but this may entail extra cost, and in this respect some hard decisions will have to be taken in the near future.

The designs of the now resistive dipole magnets for ALICE and $\mathrm{LHCb}$ have converged to what appear to be very reasonable solutions to the requirements. These magnets are also very large and will demand careful detailed (costconscious) design and follow up, but their timely procurement should not be a problem.

\section{CONCLUSION}

The LHC experiments have provided an opportunity to design magnets that push the technology clearly forward, and the whole community will benefit from these advances. In particular the discovery that one can convert the aluminium used to stabilize superconducting cable into a mechanically viable material, while maintaining a high value of RRR, paves the way for new and exciting designs of high performance magnets.

The size of the magnets has required the adoption of manufacturing and assembly techniques that were not previously familiar to magnet constructors. The large forces present have also served to concentrate the mind and search for novel solutions. The teams designing these magnet systems have risen to the challenge and are now well on the way constructing this new generation of spectrometers.

\section{REFERENCES}

[1] T. Taylor, "The choice of spectrometer magnets for LEP", Physica Scripta, Vol. 23, pp. 459-464, 1981.

[2] B. Pope, "Proposal for a high sensitivity search for muons and muon pairs using a toroidal magnet", Princeton University Publication COO3072-97, 1979.

[3] A. Hervé, "The CMS detector magnet", presented at this confererence.

[4] S. Horvarth, The CMS Conductor, presented at this conference.

[5] H. ten Kate, "The superconducting magnet system for the ATLAS detector", presented at this conference.

[6] A. Yamamoto et al., "Design and development of the ATLAS central solenoid magnet", IEEE Trans on Applied Superconductivity, vol. 9, No. 2, pp. 852-855, 1999.

[7] K. Wada, "High strength and high RRR AlNi alloy for aluminum stabilized superconductor", presented at this conference.

[8] E. Acerbi et al., "Synthesis of technological developments for the B0 model coil and the ATLAS barrel toroid coils", presented at this conference.

[9] D. E. Baynham et al., "Engineering status of the superconducting end cap toroid magnets for the ATLAS experiment at LHC", presented at this conference.

[10] F. Wittgenstein, A. Hervé, M. Feldmann, D. Luckey, I. Vetlitsky, "Construction of the L3 magnet, MT-11, Tsukuba, pp. 130-135, 1989

[11] V. Bartenev, I. Bogulslavski, V. Datskov, E. Koshurnikov, A. Shabunov, Y. Shiskov, D. Swoboda, A. Vodopianov, "Design and status of the dipole spectrometer magnet for the ALICE experiment", presented at this conference.

[12] D. George, V. Vrankovic, J. Zichy, R. Maix, "Design study of a large gap superconducting spectrometer dipole", IEEE Trans on Applied Superconductivity, vol. 9, No. 2, pp. 471-474, 1999.

[13] W. Flegel, private communication.

[14] N. Delruelle, F. Haug, G. Passardi, H. ten Kate, "The helium cryogenic system for the ATLAS experiment", presented at this conference.

[15] D. Delikaris, J.P. Dauvergne, G. Passardi, J.C. Lottin, J.P. Lottin, C. Lyraud, "The cryogenic system for the superconducting solenoid magnet of the CMS experiment", CERN LHC Project Report 165, 1998. 